EPJ Web of Conferences 59, 05015 (2013)

DOI: $10.1051 /$ epjconf/20135905015

(C) Owned by the authors, published by EDP Sciences, 2013

\title{
Nonlocal properties of ponderomotive force in strongly nonuniform high intensity laser fields
}

\author{
N. Iwata ${ }^{a}$, Y. Kishimoto and K. Imadera \\ Graduate School of Energy Science, Kyoto University, Gokasho, Uji, Kyoto 611-0011, Japan
}

\begin{abstract}
In order to study the particle dynamics in strongly nonuniform and/or localized laser fields, we extend the theory of the ponderomotive force by taking into account the laser field structure up to the higher order with respect to $\epsilon$, the ratio between the particle excursion length and the scale length of the laser field amplitude variation. This extension is realized by keeping the rigorous phase space Lagrangian structure utilizing the noncanonical Lie perturbation up to higher orders. As a result, the equations of motion in a oscillation center coordinate is derived up to the third order of $\epsilon$, which include the second and third spatial derivatives of the laser field amplitude. This denotes that the ponderomotive force depends not only on the local field gradient, but also on the curvature and its derivative. The additional higher-order force originates from the nonlocal particle motion around the oscillation center, which could have an influence especially in the case of tight focusing in the level of laser wavelength.
\end{abstract}

\section{INTRODUCTION}

The intensity of ultra-short high power lasers has reached the range of $10^{18-22} \mathrm{~W} / \mathrm{cm}^{2}$, where electrons irradiated by such lasers exhibit highly relativistic characteristics. Recently, higher intensities of $10^{23-26} \mathrm{~W} / \mathrm{cm}^{2}$ are expected by further reducing the pulse length and also the spot size to the level of laser wavelength, which will open up an entirely new scientific regime [1]. In such spatially localized laser fields, the ponderomotive force (light pressure) becomes of critical importance in determining the laser-plasma interaction [2]. The ponderomotive force has been derived by applying the averaging method to the equation of motion and is explained as the force proportional to the field gradient, which results from the first order perturbation to the uniform field [3, 4]. In this method, terms related to the higher-order derivatives in the perturbation expansion have been neglected. However, they could become important when the electromagnetic field exhibit highly localized and/or fine scale structures in space and time where the field amplitude varies within the excursion length of the particle motion.

In order to investigate the higher order effect, here, we apply the noncanonical Lie perturbation theory, which is based on the phase space Lagrangian formalism [5-7]. By introducing a noncanonical coordinate, we derive the oscillation-center equations of motion describing the ponderomotive force that includes the nonlocal effect up to the higher order corresponding to the third derivative of the field amplitude.

\section{NONCANONICAL COORDINATE TRANSFORMATION}

We consider a single particle motion in relativistic regime irradiated by transversely non-uniform high intensity laser field. We express the laser field by the normalized vector potential, $\mathbf{a} \equiv|q| \mathbf{A} / m c^{2}$, as

$$
\mathbf{a}(x, \eta)=a_{x}(x) \sin \eta \hat{\mathbf{e}}_{x}
$$

\footnotetext{
ae-mail: iwata@center.iae.kyoto-u.ac.jp
}

This is an Open Access article distributed under the terms of the Creative Commons Attribution License 2.0, which permits unrestricted use, distribution, and reproduction in any medium, provided the original work is properly cited. 
where $q$ and $m$ are the charge and rest mass of the particle, respectively, $c$ is the speed of light, $\eta \equiv \omega t-k_{z} z$ is the laser phase and $\hat{\mathbf{e}}_{x}$ is unit vector in the $x$-direction. We introduce a smallness parameter $\epsilon$ as $l / L \sim \epsilon, l^{2} / R \sim \epsilon^{2}$ and $l^{3} / T \sim \epsilon^{3}$, where $l$ is the transverse excursion length of the particle, $L^{-1} \equiv a_{x}^{-1} \partial_{x} a_{x}$ is the scale length of the gradient of the laser field amplitude, and $a_{x} / R \equiv \partial_{x}^{2} a_{x}$ and $a_{x} / T \equiv \partial_{x}^{3} a_{x}$ correspond to the field curvature and its derivative, respectively. Note that in order for the transversely nonuniform electromagnetic field in vacuum as in the present case to satisfy the Maxwell equations, a $z$-component of the vector potential is necessary in the order of $l / L$. However, we neglect it for simplicity since it is expected that the characteristics of higher order terms derived in this study are not affected qualitatively.

In the noncanonical Hamiltonian mechanics, we introduce the extended phase space expressed by canonical variables, $z_{\mathrm{c}}^{\mu}=\left(t ; \mathbf{x}, \mathbf{p}_{c}\right)$, and the corresponding covariant vector, $\gamma_{\mathrm{c} \mu}=\left(-h ; \mathbf{p}_{c}, \mathbf{0}\right)$, where $h$ is the Hamiltonian. In this paper, we use Latin indices that run from 1 to 6 whereas Greek indices run from 0 to 6 . Using these notations, the variational principle is given by $\delta \int \hat{\gamma}_{\mathrm{c}}=0$, where $\hat{\gamma}_{\mathrm{c}} \equiv \gamma_{\mathrm{c} \mu} d z_{\mathrm{c}}^{\mu}$ is referred to as a fundamental 1-form. By using the scalar relationship $\gamma_{\mathrm{c} \mu} d z_{\mathrm{c}}^{\mu}=\gamma_{\mu} d z^{\mu}$, we can obtain the variational principle and the corresponding equation of motion in the arbitrary noncanonical coordinate $z^{\mu}$ as

$$
\frac{d z^{i}}{d z^{0}}=J^{i j}\left(\frac{\partial \gamma_{j}}{\partial z^{0}}-\frac{\partial \gamma_{0}}{\partial z^{j}}\right),
$$

where $J^{i j}$ is the Poisson tensor defined as the inverse matrix of the Lagrange tensor, $\omega_{i j} \equiv \partial_{i} \gamma_{j}-\partial_{j} \gamma_{i}$. Note that, since we assume $\partial_{x} a_{x} \sim \mathcal{O}(\epsilon)$ in this study, the $x$-derivative of functions of order $\epsilon^{n-1}$ may cause terms of order $\epsilon^{n}$, e.g. $\partial_{x} \gamma_{j}^{(n-1)}=\mathcal{O}\left(\epsilon^{n-1}\right)+\mathcal{O}\left(\epsilon^{n}\right)$. Therefore, the $n-1$ th order 1 -form yields terms of both order $\epsilon^{n-1}$ and $\epsilon^{n}$ in the equation of motion.

By using the noncanonical transformation, first, we introduce a new coordinate to make the analysis easier. In the uniform laser field, the particle is known to exhibit a figure-eight oscillation in the period of $\eta$ and drift in the $z$-direction [3]. Therefore, here, we transform the independent variable from time $t$ to phase $\eta$, and also introduce the invariant of the figure-eight motion, $p_{\eta} \equiv p_{z}-\gamma m c$, as one of the coordinate variables, where $\gamma$ is the relativistic factor. The new noncanonical coordinate is defined as

$$
z_{\mathrm{c}}^{\mu} \mapsto z^{\mu}=\left(\eta ; x, y, z, p_{x}, p_{y}, p_{\eta}\right) .
$$

Here, we have also introduced the mechanical momentum, $\mathbf{p}=\mathbf{p}_{c}-m c \mathbf{a}$. The corresponding covariant vector is calculated as

$$
\gamma_{\mu}=\left(-K ; p_{x}+m c a_{x}(x) \sigma \sin \eta, p_{y}, p_{\eta}, 0,0,0\right),
$$

where $K=-\left(2 k_{z} p_{\eta}\right)^{-1}\left(m^{2} c^{2}+\mathbf{p}_{\perp}^{2}+p_{\eta}^{2}\right)$ is the new Hamiltonian and $\sigma \equiv q /|q|$ denotes the sign of the charge. We found that by taking both $\eta$ and $p_{\eta}$ as coordinate variables, the Poisson tensor is written in the same form as that in the canonical coordinate, and also the relativistic Hamiltonian can be expressed without square root, which simplifies the perturbation analysis.

\section{PERTURBATION ANALYSIS}

The Lie transformation is characterized by the near-identity transformation operator $L$ as $z^{\mu} \mapsto z^{\prime \mu}=$ $\exp (\epsilon L) z^{\mu}$. The corresponding covariant vector is transformed as $\gamma_{\mu} \mapsto \gamma_{\mu}^{\prime}=\exp (-\epsilon L) \gamma_{\mu}+\partial_{\mu} S$, where $S$ is the gauge function [5]. In the Lie perturbation method, we utilize the degrees of freedom of the gauge and transformation generator in order to describe the perturbed motion simply. In this study, we take the $n$ th-order generator, covariant vector and gauge function to satisfy $g^{(n) 0}=0, \gamma_{i}^{\prime(n)}=0$, and $\overline{S^{(n)}}=0$, respectively, where overline indicates the average over $\eta$-period fast oscillations. 
Here, we expand the amplitude of the laser field given by Eq. (1) around the oscillation center of the figure-eight orbit, $X$, as

$$
a_{x}(x)=a_{x}(X)\left[1+\epsilon \frac{\tilde{x}}{L}+\epsilon^{2} \frac{\tilde{x}^{2}}{2 ! R}+\epsilon^{3} \frac{\tilde{x}^{3}}{3 ! T}+\cdots\right],
$$

where $\tilde{x} \equiv X-x$, and $L, R$ and $T$ are evaluated at $X$. In the zeroth order of $\epsilon$, Eq. (5) denotes a uniform laser field. In this order, we treat $a_{x}(X)$ as a constant. Then, from the zeroth order 1-form, $\hat{\gamma}^{(0)}=-K d \eta+p_{x}^{(0)} d x^{(0)}+p_{y}^{(0)} d y^{(0)}+p_{\eta}^{(0)} d z^{(0)}$, we can derive the equations of motion which yield the figure-eight motion in the uniform field, and the conservation relation, $d p_{\eta}^{(0)} / d \eta=0$. Here, we take the initial condition $\left(\mathbf{x}, \mathbf{p}_{\perp}, p_{\eta}\right)=\left(\mathbf{x}_{\perp 0}, 0,0,0,-m c \zeta_{0}\right)$ and $p_{z}=p_{z 0}$ at $\eta=0$. In this notation, $\zeta_{0}=1$ when the initial momentum of the charged particle is zero, i.e., $p_{z 0}=0$. The excursion length $l$ is then obtained as $l(X)=a_{x}(X) / k_{z} \zeta_{0}$. Next, by using the zeroth-order solution, we consider another noncanonical transformation from the coordinate $z^{\mu}$ to that of the oscillation center, $Z^{\mu}=\left(\eta ; X, Y, Z, P_{x}, P_{y}, p_{\eta}\right)$. The relation between the old and new coordinates are given by

$$
\begin{gathered}
x(X, \eta)=X+\sigma l(X) \cos \eta, \\
z(X, Z, \eta)=Z-\frac{k_{z} l^{2}(X)}{8} \sin 2 \eta, \\
p_{x}\left(X, P_{x}, \eta\right)=P_{x}-\sigma m c a_{x}(X) \sin \eta,
\end{gathered}
$$

$y(Y)=Y, p_{y}\left(P_{y}\right)=P_{y}$ and $p_{\eta}\left(p_{\eta}\right)=p_{\eta}$, where the second terms on the right-hand side of Eqs. (6)-(8) are the oscillatory part of the zeroth order orbit. Note that, here, we treat $a_{x}(X)$ not as a constant but as a variable depending on $X$, although hereafter we simply express it as $a_{x}$.

Based on the coordinate $Z^{\mu}$, we derive the equations of motion for perturbed trajectory of the oscillation center. Here, we consider the Lie transformation $Z^{\mu} \mapsto Z^{\prime \mu}=\left(\eta ; X^{\prime}, Y^{\prime}, Z^{\prime}, P_{x}^{\prime}, P_{y}^{\prime}, p_{\eta}^{\prime}\right)$ up to the third order with respect to $\epsilon$. The covariant vector in the coordinate $Z^{\prime \mu}$ is determined as

$$
\begin{gathered}
\Gamma_{0}^{\prime(0)}=-K-(1+\alpha) P_{x}^{\prime} \sigma l \sin \eta+p_{\eta}^{\prime} \frac{k_{z} l^{2}}{4}\left[\alpha^{2}-\left(1+\alpha^{2}\right) \cos 2 \eta\right], \\
\Gamma_{0}^{\prime(2)}=\epsilon^{2} \frac{l}{16} p_{\eta}^{\prime} k_{z} l\left(A \frac{l^{2}}{R}+B \frac{l^{2}}{L^{2}}\right)+\epsilon^{2} \frac{P_{x}^{\prime 2}}{p_{\eta}^{\prime} k_{z}}\left[\frac{1}{2}(1+\alpha) \frac{l^{2}}{R}-\left(\alpha+\frac{1}{4}\right) \frac{l^{2}}{L^{2}}\right],
\end{gathered}
$$

$\Gamma_{0}^{\prime(1)}=\Gamma_{0}^{\prime(3)}=0$ and $\Gamma_{i}^{\prime}=\left(P_{x}^{\prime}, P_{y}^{\prime}, p_{\eta}^{\prime}, 0,0,0\right)$, where $\alpha \equiv m c \zeta_{0} / p_{\eta}^{\prime}, A=-2 \alpha^{3}+2 \alpha+1, B=-4 \alpha^{3}-$ $5 \alpha^{2} / 2+4 \alpha+7 / 4$ and $K$ has the same functional form as in the old coordinate. Note that due to the restriction to the Lie transformation $\overline{S^{(n)}}=0$, no secular term is added from the backward Lie transformation. Thus, the secular motion of the oscillation center is determined by the 1-form $\hat{\Gamma}^{\prime}$ up to the order of $\epsilon^{3}$. Since $\Gamma_{\mu}^{\prime}$ has no $Y^{\prime}$ - and $Z^{\prime}$-dependence, the corresponding variables found to be constant, i.e., $d P_{y}^{\prime} / d \eta=0$ and $d p_{\eta}^{\prime} / d \eta=0$. Then, the equations of motion up to the third order in the transverse direction become

$$
\begin{gathered}
\frac{d X^{\prime}}{d \eta}=\frac{P_{x}^{\prime}}{m c \zeta_{0} k_{z}}\left(1+\epsilon^{2} \frac{3}{2} \frac{l^{2}}{L^{2}}\right) \\
\frac{d P_{x}^{\prime}}{d \eta}=-\frac{m c a_{x}}{2}\left[\epsilon \frac{l}{L}+\frac{\epsilon^{3}}{8}\left(\frac{3}{2} \frac{l}{L} \frac{l^{2}}{R}+\frac{l^{3}}{T}-\frac{3}{2} \frac{l^{3}}{L^{3}}\right)\right]+\epsilon m c a_{x} \frac{l}{L} \cos 2 \eta
\end{gathered}
$$

These equations determine the transverse secular motion of the oscillation center. In the first term on the right-hand side of Eq. (12), the third-order terms related to $R^{-1}$ and $T^{-1}$ are found to appear in addition to the first-order ponderomotive force proportional to the field gradient $a_{x} / L$. This result indicates that 

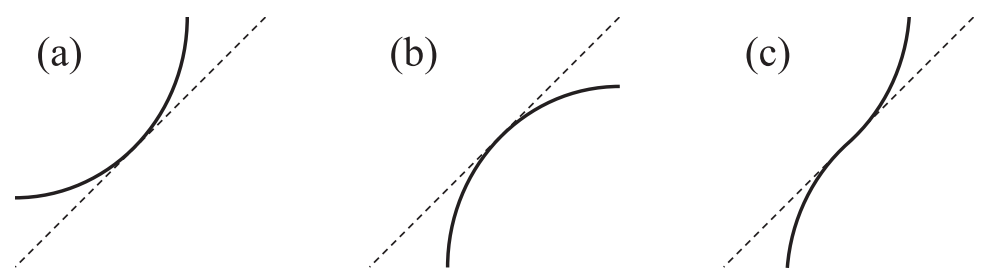

Figure 1. Laser field amplitude with (a) positive curvature, (b) negative curvature, and (c) positive third derivative.

the oscillation center of the particle is influenced not only by the local gradient of laser field amplitude, but also by the curvature and its derivative, which corresponds to the higher order nonlocal orbit effects to the particle motion. This feature is explained from Fig. 1 representing three laser fields where the amplitudes exhibit (a) concave and (b) convex shapes and (c) around an inflection point. In the cases of (a) and (b), the local gradients are same at the center points. However, since the curvatures are different, i.e. positive in (a) whereas negative in (b), the resultant ponderomotive force is enhanced in (a) whereas reduced in (b). The case (c) is found to enhance the ponderomotive force since the third derivative is positive. The other feature in Eq. (12) is the term proportional to $\cos 2 \eta$ that is the order $\epsilon$. Although this term exhibits an oscillation, it can cause a secular motion in the third order $\epsilon^{3}$ via the coupling with the oscillatory component in $a_{x}(X)$ and $l(X)$. It is interesting to note that the curvature term appears as the product with the local gradient, i.e. $l^{3} / R L$, leading to the third order, but not alone as $l^{2} / R$. This results from the symmetric nature of curvature, i.e., the nonlocal effect is cancelled during a laser period. The similar parity relation that the even derivatives do not appear alone in the equation of motion has been confirmed in the present Hamiltonian formalism. Note here that, in Eqs. (11) and (12), the usual relation between position and momentum is not held due to the second term in the bracket on the right-hand side of Eq. (11). The usual relation is recovered after executing the backward Lie transformation.

\section{SUMMARY}

Based on the noncanonical Lie perturbation theory, we investigated the higher order terms to the ponderomotive force in high intensity laser fields. Introducing a noncanonical oscillation-center coordinate, we derived the equations of motion which determine the secular motion of the oscillation center up to the third order of $\epsilon$ (expansion parameter). In the third order, the terms corresponding to the second- and third-derivatives are added to the conventional first-order ponderomotive force. These results indicate that the oscillation center trajectory is determined not only by the local field gradient but also by the local curvature and its derivative as shown in Eqs. (11) and (12) due to the nonlocal particle motion around the oscillation center. Those higher order terms related to the higher-order field structure could become important in determining the laser-particle interaction specifically in strongly nonuniform laser fields in the order of laser wavelength.

In this study, we have assumed the vector potential Eq. (1) and neglected the next order $a_{z}$ component as discussed in Sec. 2. Here, it is found that the $a_{z}$ component affects the ponderomotive force in the second order as a term proportional to $\epsilon^{2} a_{z} l / L$, which is considered to be the effect associated with the change of the transverse $E_{x}$ field in the moving frame. In the third order $\epsilon^{3}$, a term proportional to $\epsilon^{3} a_{x} a_{z} l^{2} / R$ is considered, however, it is expected to disappear due to the symmetric nature of curvature. The other is a term proportional to $\epsilon^{3} a_{x} a_{z} l^{2} / L^{2}$, which essentially denotes a local effect. Thus, the $a_{z}$ component is expected not to affect the characteristics of the present result qualitatively. Here, we choose the phase $\eta=\omega t-k_{z} z$ as the independent variable, which is left unaffected up to higher orders. However, when we consider a transversely focused laser with a finite $\mathbf{k}_{\perp}$, it suffers a phase shift around the focal point [4], which may also affect the higher order dynamics. We will study this point as a future work. 


\section{References}

[1] G. A. Mourou, T. Tajima and S. V. Bulanov, Rev. Mod. Phys. 78, 309 (2006)

[2] E. Esarey, C. B. Schroeder and W. P. Leemans, Rev. Mod. Phys. 81, 1229 (2009)

[3] P. Gibbon, Short Pulse Laser Interactions with Matter (Imperial College Press, London, 2005) pp. 31-39

[4] B. Quesnel and P. Mora, Phys. Rev. E 58, 3719 (1998)

[5] J. R. Cary and R. G. Littlejohn, Ann. Phys. 151, 1 (1983)

[6] N. Iwata, K. Imadera and Y. Kishimoto, Plasma Fusion Res. 5, 028 (2010)

[7] N. Iwata, Y. Kishimoto and K. Imadera, Plasma Fusion Res. 6, 2404105 (2011) 